\title{
Spatio-temporal patterns of bacteria caused by collective motion
}

\author{
So Kitsunezaki* \\ Dep. of Physics, Graduate School of Human Culture, Nara Women's University, \\ Nara, 630-8506, Japan
}

\begin{abstract}
In incubation experiments of bacterial colonies of Proteus Mirabilis, collective motion of bacteria is found to generate macroscopic turbulent patterns on the surface of agar media. We propose a mathematical model to describe the time evolution of the positional and directional distributions of motile bacteria in such systems, and investigate this model both numerically and analytically. It is shown that as the average density of bacteria increases, non-uniform swarming patterns emerge from a uniform stationary state. For a sufficient large density, we find that spiral patterns are caused by interactions between the local bacteria densities and the rotational mode of the collective motion. Unidirectional spiral patterns similar to those observed in experiments appear in the case in which the equilibrium directional distribution is asymmetric.
\end{abstract}

Key words: collective motion, spatio-temporal patterns, unidirectional spirals, bacterial colonies, Proteus Mirabilis PACS: 05.45.-a, 87.18.Ed, 87.18.Hf

\section{Introduction}

In systems of self-propelled particles, collective motion is known to appear with an increase in population through a kind of phase transition [1-3]. Interactions between modes of collective motion and local densities often result in macroscopic spatio-temporal patterns [4]. Traffic jams and schools of fish are well-known examples. Bacterial colonies represent one type of system that can be studied experimentally in which very large populations of individual

\footnotetext{
* Tel.: +81 74220 3988; fax: +81742 203988 .

Email address: kitsune@ki-rin.phys.nara-wu.ac.jp (So Kitsunezaki).
} 
self-propelled elements can exist $[5,6]$. Bacterial colonies have a wide variety of morphologies, with that appearing in any given case depending on the species and the conditions of the culture [7-11]. Although most such patterns are essentially static after they have formed near the growing interface of the colony, spatio-temporal patterns, such as turbulent patterns and spiral patterns, have been reported to appear in experiments employing Proteus Mirabilis [12-14], and the collective motion of swarming bacteria have been observed microscopically in such colonies. These spatio-temporal patterns resemble those observed in other dissipative systems, such as reaction-diffusion systems and liquid crystals [15]. We are interested in the mechanism of collective motion that causes such patterns. In this study we propose a mathematical model to describe the time evolution of the positional and directional distributions of bacteria.

Here we briefly summarize the main results of experiments studying proteus [12-14]. Proteus is a rod-shaped bacterium whose motility is made possible by many flagella protruding from its surface. Its length is approximately 10 $\mu \mathrm{m}$, and it moves at a speed of $20-30 \mu \mathrm{m} / \mathrm{s}$ on the surface of agar [16-18]. Although Proteus is famous for producing concentric-ring-like colonies, spatiotemporal patterns are observed on different culture conditions in which agar is softer and more nutrient-rich. They appear after a colony has spread over the surface of its enclosed environment and exist for several hours. These patterns have the following features.

(F1) They are not patterns of bacteria density, but of the local directional order of bacteria. These patterns can be observed by exploiting the heterogeneity of certain optical properties. The bacteria density is approximately uniform in typical spiral patterns. (F2) Patterns appear as the bacteria density gradually increases. In these experiments, the cultures are nutrient-rich. (F3) Targets and spirals are occasionally formed in these patterns. The spirals rotate periodically in time. The characteristic width of stripes is $2-3 \mathrm{~mm}$, and the period is $1-2 \mathrm{~min}$. (F4) Only counterclockwise spirals are observed. This is believed to have some biological cause.

The features (F2) and (F3) suggest that the growth of the bacteria population is slow compared with the characteristic time of the pattern evolution, and that a lack of nutrients plays no role in these phenomena. For these reasons, we ignore the effects of both nutrient concentration and bacterial multiplication and consider only kinetic properties of bacteria in our model.

In this paper, we first construct a 1-dimensional model. We introduce local equilibrium distributions of bacteria phenomenologically to describe interactions among bacteria as a kind of mean field. The results of numerical simulations and a linear stability analysis are reported briefly. In Section 3, we extend this model to 2-dimensional systems. Owing to the freedom of rotation, the behavior of the system becomes much more complex. Unidirectional spiral patterns can be caused to form by adopting an asymmetric equilibrium directional distribution. In the case that the population density is sufficiently large, 
simpler equations are derived from our model using a reductive perturbation method. We summarize the results in Section 4.

\section{1-dimensional model}

In this section we construct a phenomenological model consisting of equations that describe the situation in which two groups of bacteria move in opposite directions in a 1-dimensional system. We treat the total number of bacteria as a fixed parameter, because we ignore population growth, as mentioned in the Introduction.

It is known that a single bacterium in water exhibits repeated uniform linear motion punctuated by random changes of direction [19]. However, in the case of bacteria densely populating the surface of a culture medium, quantitative features of their motion have not been investigated thoroughly. Short-range interactions among individuals are very complicated due to many-body collisions, hydrodynamic effects, tangles of flagella and biological communication. Therefore, assuming that, as in water, bacteria in a culture medium move linearly with an approximately constant speed $v$ and change their direction randomly with a time-independent probability $\gamma_{0}$, we introduce local equilibrium distributions to describe interactions as a kind of mean field. Local interactions among assemblages moving in various directions are described as relaxation to the equilibrium states, as in the collision interval theory for the Boltzmann equation.

We express the densities of bacteria moving in the positive and negative directions along the $x$ axis at time $t$ as $n_{+}(x, t)$ and $n_{-}(x, t)$ and the fluxes as $j_{+}(x, t)$ and $j_{-}(x, t)$, respectively. The local average density is defined as $\rho(x, t) \equiv\left\{n_{+}(x, t)+n_{-}(x, t)\right\} / 2$, and we introduce the order parameter quantifying collective motion as $W(x, t) \equiv\left\{n_{+}(x, t)-n_{-}(x, t)\right\} / 2$. Expressing the local equilibrium distributions as $\rho g_{ \pm}$, the continuity equations for $n_{ \pm}$are given by

$$
\frac{\partial n_{+}}{\partial t}+\frac{\partial j_{+}}{\partial x}=\gamma_{0}\left(g_{+} \rho-n_{+}\right), \quad \frac{\partial n_{-}}{\partial t}+\frac{\partial j_{-}}{\partial x}=\gamma_{0}\left(g_{-} \rho-n_{-}\right) .
$$

The second term of each equation yields the property that the system tends to approach local equilibrium states through changes of direction.

The fluxes are expected to decrease as "traffic jams" occur in dense states. We assume that the functions $j_{ \pm}(x, t)$ depend on the lowest order of the density 
gradient according to

$$
j_{ \pm}= \pm v n_{ \pm}-D \frac{\partial n_{ \pm}}{\partial x}
$$

The second term on the right-hand side acts as a diffusion term and prevents the emergence of numerical instabilities in the model. We conjecture that such diffusion is caused by short-range interactions among bacteria, such as collisions. We note that random changes of direction also result in diffusive behavior with a diffusion coefficient of the order of $v^{2} / \gamma_{0}$ even if this term were not included.

The equilibrium distributions are expected to be different for the two groups, as we anticipate the appearance of collective motions. We assume that $g_{ \pm}$are functions of the order parameter $W$ and that $g_{+}=g_{-}$for $W=0$. Stipulating the conservation of total number and the reflection symmetry of Eqs. (1) and (2), we obtain the relations $g_{+}(W)+g_{-}(W)=2$ and $g_{-}(W)=g_{+}(-W)$. Hence, $g_{+}(W)-1$ is an odd function of $W$. In this study we assume it to be a third-order function of the following form:

$$
g_{+}(W)=g_{-}(-W)=g_{1}(a W), \quad g_{1}(z) \equiv 1+\left(1-b z^{2}\right) z
$$

Here $b$ is a positive constant, and $a$ is a constant corresponding to the coefficient of the linear order term. We assume this form for convenience with regard to scaling, as discussed below.

We choose $v, D$ and $a$ to be 1 , without loss of generality, as this can be accomplished by scaling $t, x$ and $n_{ \pm}$. Equations (1), (2) and (3) can be written in the form of two equations for $\rho$ and $W$ :

$$
\begin{aligned}
\frac{\partial \rho}{\partial t} & =-\frac{\partial W}{\partial x}+\frac{\partial^{2} \rho}{\partial x^{2}}, \\
\frac{\partial W}{\partial t} & =\frac{1}{\tau}\left(\rho-1-b \rho W^{2}\right) W-\frac{\partial \rho}{\partial x}+\frac{\partial^{2} W}{\partial x^{2}} .
\end{aligned}
$$

Here we have used $\tau \equiv v^{2} / \gamma_{0} D$. Note that the spatial average of the density, $\bar{\rho} \equiv(1 / L) \int_{0}^{L} d x \rho(x, t)$, is treated as a parameter, because it is conserved in time.

The behavior of solutions of Eq. (4) can be guessed from the uniform solutions and their stabilities. As $\bar{\rho}$ increases, the trivial stationary solution $(\rho, W)=(\bar{\rho}, 0)$ destabilizes at $\bar{\rho}=1$. Then, considering only uniform solutions, the trivial solution branches into the two traveling solutions $(\rho, W)=$ $(\bar{\rho}, \pm \sqrt{(\bar{\rho}-1) / b \bar{\rho}})$. The eigenvalues obtained from the linear stability anal- 

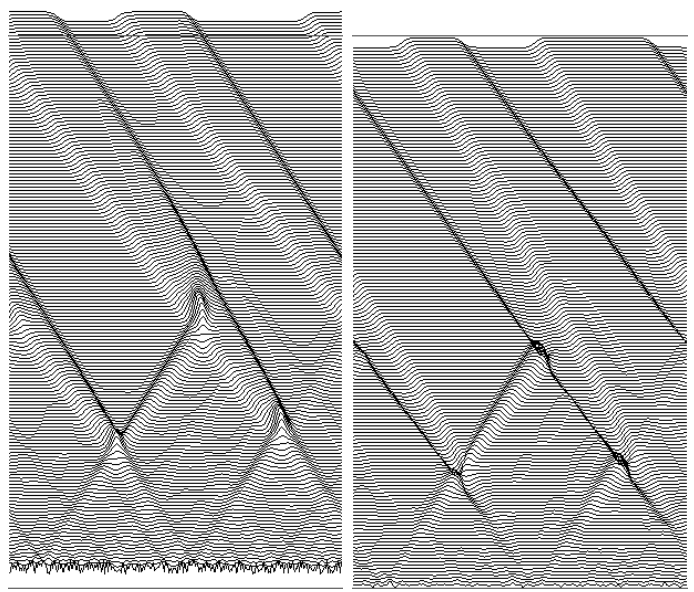

Fig. 1. Space-time plot of $\rho$ (left) and $W$ (right) obtained from a numerical simulation with $\bar{\rho}=1.1$.

ysis are

$$
\begin{array}{lr}
\lambda=\epsilon / 2-k^{2} \pm \sqrt{\epsilon^{2} / 4-k^{2}} & \text { for the trivial solution, } \\
\lambda=-\epsilon-k^{2} \pm \sqrt{\epsilon^{2}-k^{2}-i k W / \tau \bar{\rho}} & \text { for the traveling solutions, }
\end{array}
$$

where $k$ is the wave number and $\epsilon \equiv(\bar{\rho}-1) / \tau$. Both eigenvalues given in Eq. (5a) have positive real parts for small $k$ when $\bar{\rho}$ increases beyond 1 . Therefore, the emergence of collective motion in this model does not appear through a pitchfork-type bifurcation. We conjecture that nonuniform solutions appear, because the uniform traveling solutions are unstable for $4 \tau b \bar{\rho}^{3}(2 \epsilon+1) \epsilon<1$. However, for large $\bar{\rho}$, the system is expected to settle into either of the two uniform traveling states.

We carried out numerical simulations for a system of size $L=150$ with $\tau=$ $b=1$. The Euler method was used, with spatial mesh size $\Delta x=0.5$ and time step $\Delta t=0.01$. Periodic boundary conditions were employed, and slightlyperturbed uniform states were prepared as the initial states by adding smallamplitude white noise to the trivial solution, $(\rho, W)=(\bar{\rho}, 0)$. Figures 1 and 2 display the results for $\bar{\rho}=1.1$ and $\bar{\rho}=2.2$, respectively. In each spacetime plot, the abscissa corresponds to the $x$ coordinate, and the ordinate corresponds to time, with the series of plots moving up the ordinate axis representing data obtained every three time units. As we hypothesized above, nonuniform traveling solutions appear as $\bar{\rho}$ increases. Then, as $\bar{\rho}$ increases further, uniform collective motion emerges.

We next investigated the spatial average of the flux, $\overline{W-\partial \rho / \partial x}=\bar{W}$, changing $\bar{\rho}$ quasi-statically. Figure 3 plots the absolute value of $\bar{W}$ with the solid curve. In this simulation, the small term $c \rho$ is added to the right-hand side of 


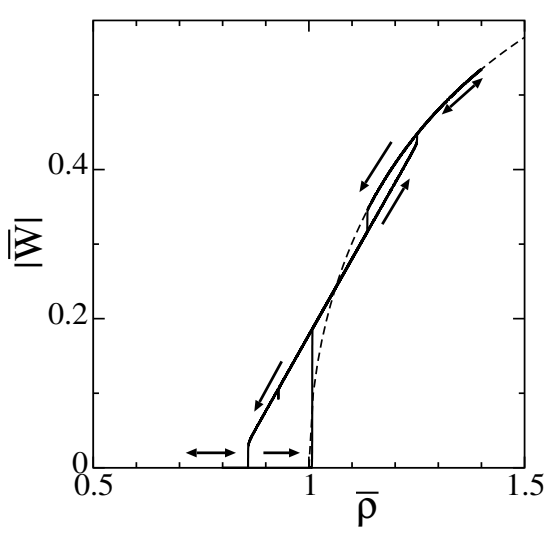

Fig. 3. Dependence of the average flux on $\bar{\rho}$.

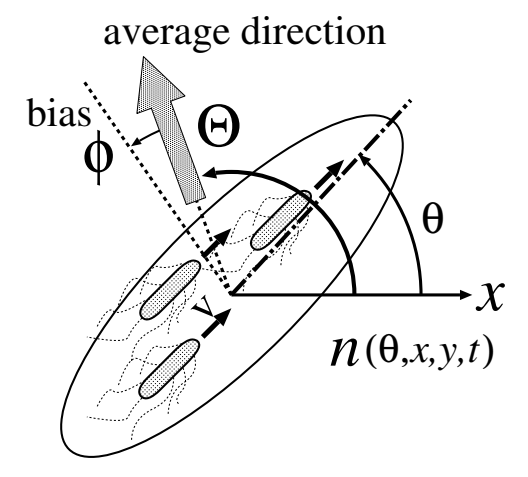

Fig. 4. 2-d model.

Eq. (4a), and $\bar{\rho}$ is increased from 0.8 to 1.4 and then decreased to 0.8 . Here we used $c= \pm 2 \times 10^{-6}, L=125$ and $\Delta x=0.25$. The dotted curve corresponds to $|W|$ for the uniform traveling states. The hysteresis exhibited in Fig. 3 is caused by the appearance of non-uniform traveling states.

\section{2-dimensional model}

We extend our model to 2 -d systems in this section. Here, the function $n(\theta, \boldsymbol{x}, t)$ represents the local density of bacteria moving in the direction of the angle $\theta$ at the position $\boldsymbol{x} \equiv(x, y)$ and time $t$. The local average density $\rho(\boldsymbol{x}, t)$ and the complex order parameter $W(\boldsymbol{x}, t)$ are defined as $\rho \equiv\langle n\rangle$ and $W \equiv$ $\left\langle n e^{i \theta}\right\rangle \equiv|W| e^{i \Theta}$, using the directional average $\langle\cdots\rangle \equiv(1 / 2 \pi) \int_{0}^{2 \pi} d \theta \cdots$. The magnitude and direction of collective motion are characterized by $|W|$ and $\Theta$. Equation (1) is naturally extended to the equation

$$
\frac{\partial n}{\partial t}+\nabla \cdot \boldsymbol{j}=\gamma_{0}\left(g_{2} \rho-n\right)
$$

where $g_{2} \rho$ denotes the local equilibrium distribution. As in Sec. 2, we assume that $g_{2}$ is a function of $W$ and $\theta$ and that it is constant for $W=0$. Also, $g_{2}$ must be a function of the single variable $W e^{-i \theta}$ in order for Eq. (6) to possess rotational symmetry. Stipulating the conservation of the total number imposes the condition $\left\langle g_{2}\right\rangle=1$. We express $g_{2}$ as $g_{2}(W, \theta) \equiv g(z)$, using the quantity $z \equiv a W e^{-i \theta}$. The constant $a$ is generally a complex number. It represents the coefficient of the linear-order term of $g(z)$ assumed below, as in the 1-d model.

The flux in the direction of the angle $\theta$ is expressed in forms of the velocity vector $\boldsymbol{v} \equiv v(\cos \theta, \sin \theta)$, using an equation similar to Eq. (2),

$$
\boldsymbol{j}=\boldsymbol{v}\left(1-\frac{D}{v^{2}} \boldsymbol{v} \cdot \nabla\right) n .
$$


Through an appropriate scaling of $t, \boldsymbol{x}$ and $n$, the parameters $v$ and $D$ are set to 1 , and $a$ is expressed as $a \equiv e^{i \phi}$. Then, Eqs. (6) and (7) can be combined into the following:

$$
\begin{array}{r}
\frac{\partial n}{\partial t}=\frac{1}{\tau}\{\rho g(z)-n\}-\nabla \cdot \boldsymbol{v} n+(\nabla \cdot \boldsymbol{v})^{2} n, \\
z \equiv W e^{i(\phi-\theta)}=|W| e^{i(\Theta+\phi-\theta)} .
\end{array}
$$

Considering the Fourier expansion of $g(z)$ with respect to $\theta$, we adopt the simplest function containing only the lowest-order terms in $e^{ \pm i \theta}$,

$$
g(z) \equiv 1+2\left(1-b|z|^{2}\right) \operatorname{Re} z=1+2|W|\left(1-b|W|^{2}\right) \cos (\Theta+\phi-\theta),
$$

where $b$ is assumed to be a positive real number. The term nonlinear in $|W|$ is necessary to prevent the divergence of solutions when the trivial uniform state becomes unstable.

For given $\rho>\rho_{c}$, this system has the uniform solution

$$
\begin{aligned}
n & =\rho+2|W| \cos (\Theta-\theta) \\
|W|=\sqrt{\frac{\rho-\rho_{c}}{b \rho}}, \quad \Theta=\omega t+\text { const. }, \quad \rho_{c} & \equiv \frac{1}{\cos \phi}, \quad \omega \equiv \frac{\tan \phi}{\tau} .
\end{aligned}
$$

Here, $n$ is periodic in time if $\phi \neq 0$, and its directional distribution is symmetric with respect to $\theta=\Theta$. This solution becomes stable as $\rho-\rho_{c}$ increases. The quantities $\Theta$ and $\rho$ are not determined locally, and they are expected to interact as slow modes through the spatial derivative terms [20-22].

We next compare the two cases $\phi=0$ and $0<|\phi| \ll 1$. In the latter case, the collective motion rotates gradually in the direction determined by the sign of $\phi$. In fact, some experiments show that colonies grown radially from an incubated point form shapes with chirality whose direction is determined uniquely for each species. This is believed to be caused by some biological factor, such as the rotation direction of the flagella $[8,23]$.

We carried out numerical simulations of Eqs. (8) and (9) using the Euler method. The step size of $\theta, \boldsymbol{x}$ and $t$ were $\Delta \theta=2 \pi / 16, \Delta x=2$ and $\Delta t=0.01$. Periodic boundary conditions were employed with a system size $L=400$. The initial conditions were taken as $n(\theta, \boldsymbol{x}, 0)=\bar{\rho}\{1+\xi(\theta, \boldsymbol{x})\}$, using smallamplitude white noise $\xi$, satisfying $|\xi|<10^{-4}$. We chose the parameter values $\tau=0.1$ and $b=1$. Figures 5 and 6 display results for the two cases $\phi=0$ and $\phi=0.02$. For each case, snapshots obtained from two simulations with $\bar{\rho}=1.2$ and 5.0 are presented at the top and bottom of the figure. The scales used for $\rho$ and $W$ are displayed in Fig. 7. As in the 1-d model, the system settles into the trivial uniform state with $W=0$ for $\bar{\rho}<\rho_{c}$, and collective motion begins to appear as $\bar{\rho}$ increases. Spatial variations of both $\rho / \bar{\rho}$ and $|W|$ increase in size as $\left(\bar{\rho}-\rho_{c}\right) / \rho_{c}$ becomes small. In particular, in the case $\phi \neq 0$, 
several localized regions exhibiting vortex motion appear, and these regions slowly change shape.

In contrast to the 1-d model, this system can have topological defects in the $\Theta$ field, and non-uniform states are observed even when $\bar{\rho}$ is sufficiently large compared with $\rho_{c}$. Spirals grow around these defects for $\phi \neq 0$, while patterns are practically frozen for $\phi=0$, except for the very slow motion of defects. The spiral patterns are similar to those reported in experiments. However, both clockwise and counterclockwise spirals grow initially in these spiral patterns. This differs from the experimentally observed behavior referred to as (F4) in the Introduction.

Unidirectional spiral patterns are observed when we adopt the asymmetric equilibrium distribution with respect to $\theta=\Theta+\phi$ represented by

$$
g(z)=1+2\left(1-b|z|^{2}\right)\left\{\operatorname{Re}(z)+c \operatorname{Im}\left(z^{2}\right)\right\}
$$

instead of Eq. (9). The imaginary part of $z^{2}$ gives a term that is second order in $e^{ \pm i \theta}$. We are not interested in the real parts of $z^{2}$ because it does not break the symmetry. Although there are presently no experimental data verifying this distribution, it is plausible that equilibrium distributions lose symmetry in the case that the collective motion deviates from a straight line in a particular direction. Assuming Eq. (11) and the condition $\phi \ll 1$, the nontrivial uniform solution becomes asymmetric with respect to $\theta=\Theta$, taking the form

$$
n \simeq \rho+2|W| \cos (\Theta-\theta)+2 c|W|^{2} \sin 2(\Theta-\theta)+O\left(\phi^{2}\right),
$$

while the equation for $W$ is the same as Eq. (10b).

Figure 8 depicts the result of a numerical simulation with $c=-0.5$. In the case that the equilibrium distribution is sufficiently asymmetric with respect to the direction of collective motion, only spiral patterns with rotation in one of the directions can grow. In order to investigate the growth of spirals, we carried out simulations using the special initial conditions, $n(\theta, x, y, 0)=\bar{\rho}\{1+$ $\left.2 R e\left(W_{0} e^{-\theta i}\right)\right\}$ and $W_{0}=-0.1\{\cos (2 \pi y / L)+i \cos (2 \pi x / L)\}$. This initial state contains four topological defects. Figure 9 displays snapshots of $W$ obtained at $t=0,500$ and 2500 for the two cases $c=0$ and $c=-0.5$. For both values of $c$, we find that as the system evolves, eventually one of the two types of spirals dominates. In the case $c=0$, both types of spirals grow initially, but eventually one type begins to shrink while the other continues to grow [24]. However, when $|c|$ is large, even at early times, before there can be any interaction between spirals, only one type is able to form.

Assuming that $\tau$ and $\phi$ are sufficiently small, we can reduce the 2-d model to equations for $\rho$ and $\Theta$ using the reductive perturbation method, as described in Appendix A. These equations accurately describe the propagation of the spirals, although the behavior of the spiral cores may not be faithfully reflected. 

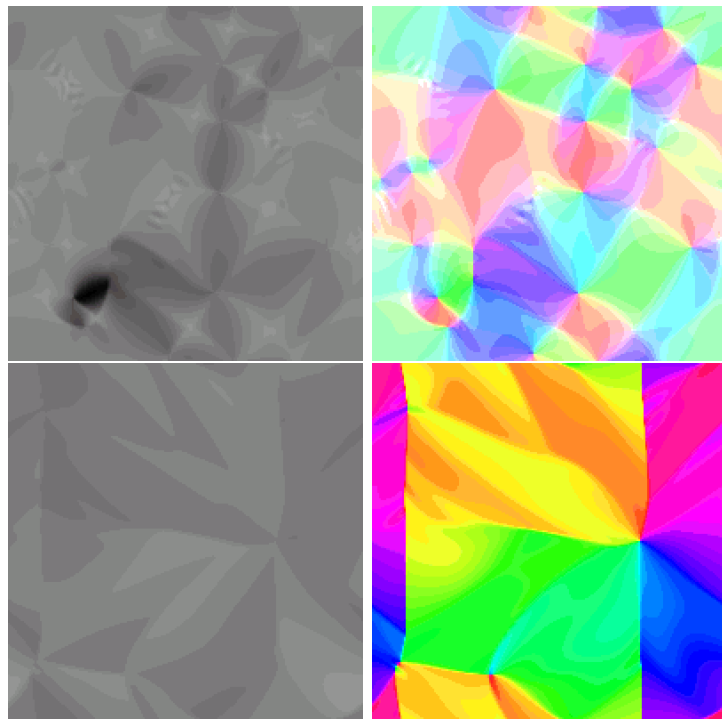

Fig. 5. Snapshots at $t=2500$ obtained from simulations with $\phi=0, \bar{\rho}=1.2$ (top) and 10.0 (bottom). $\rho$ and $W$ are displayed on the left and right, respectively.

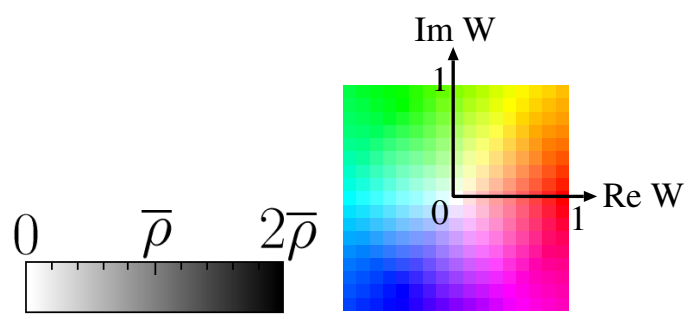

Fig. 7. The scales used for $\rho$ and $W$.

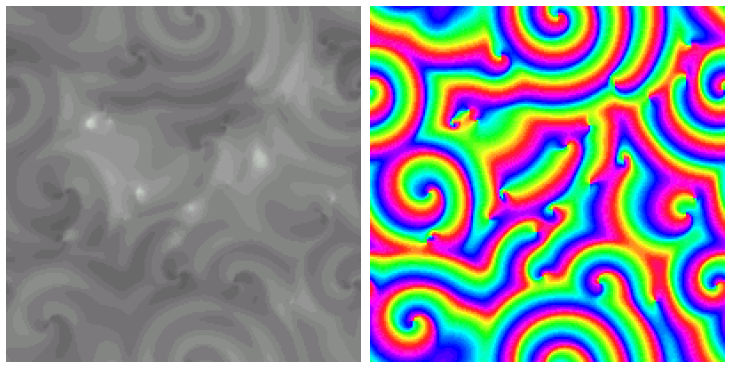

Fig. 8. Snapshots obtained from a numerical simulation using Eq. (11) with $c=-0.5$. The other parameters are the same as in the case of the bottom snapshots of Fig. 6 .

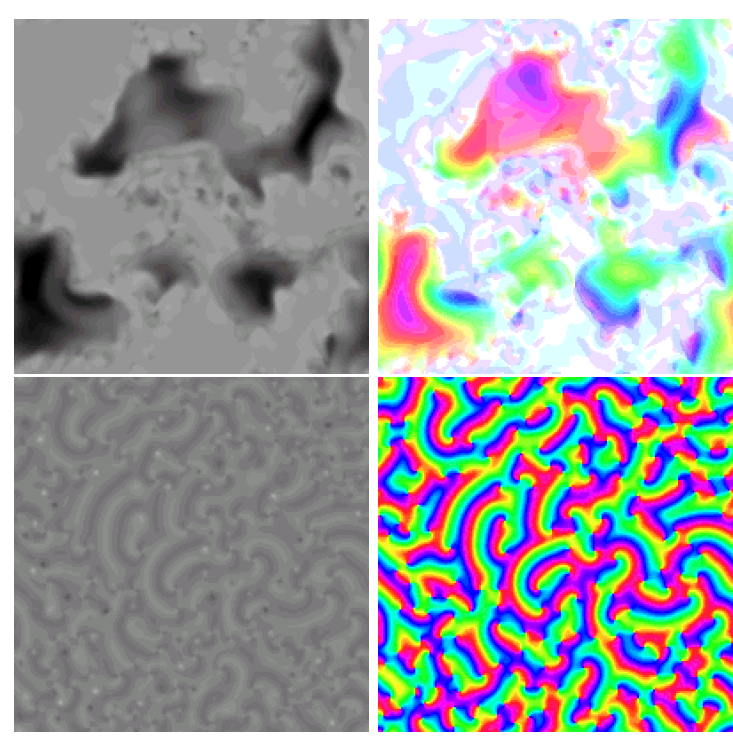

Fig. 6. Snapshots at $t=2500$ obtained from numerical simulations with $\phi=0.02$, $\bar{\rho}=1.2$ (top) and 10.0 (bottom).

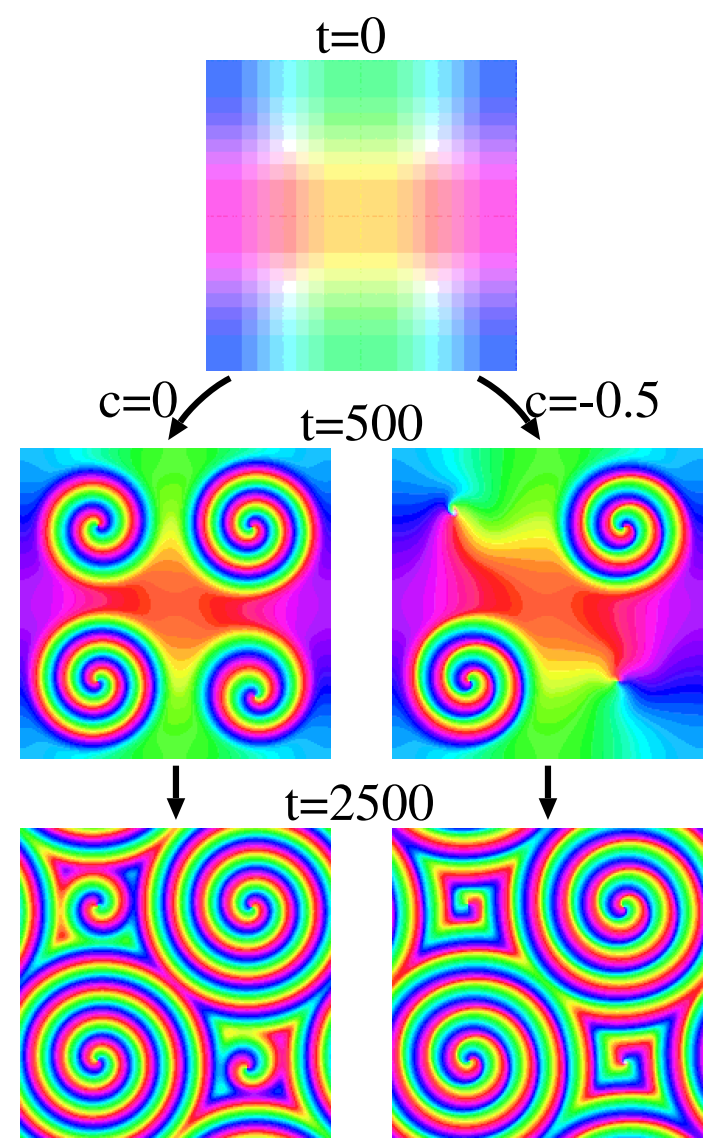

Fig. 9. Growth of spirals for the two cases $c=0$ (left) and $c=-0.5$ (right). Here we have $\phi=0.02$ and $\bar{\rho}=5.0$. 

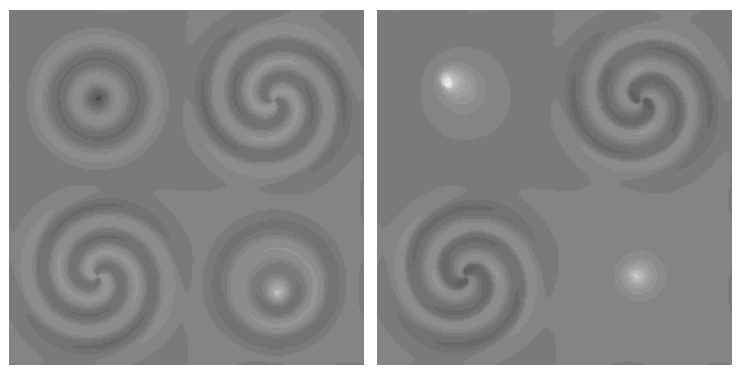

Fig. 10. Snapshots of $\rho$ at $t=500$ for the simulations depicted in Fig. 9.

In regions that $\rho$ is sufficiently larger than $\rho_{c}$, the reduced equations (A.6) and (A.7) are approximated by the following:

$$
\begin{aligned}
\frac{\partial \tilde{\rho}}{\partial t} & =\frac{1}{2} \triangle \tilde{\rho}-\partial_{\perp} \Theta+\tilde{c}\left\{\left(\partial_{/ /}^{2}-\partial_{\perp}^{2}\right) \Theta+2\left(\partial_{/ /} \Theta\right)\left(\partial_{\perp} \Theta\right)\right\}, \\
\frac{\partial \Theta}{\partial t} & =\omega+\frac{1}{2} \triangle \Theta-\frac{1}{2} \partial_{\perp} \tilde{\rho}+\frac{1}{4}\left(\partial_{\perp}^{2}-\partial_{/ /}^{2}\right) \Theta+\tilde{c} \partial_{\perp} \Theta, \\
\partial_{/ /} & \equiv \cos \Theta \frac{\partial}{\partial x}+\sin \Theta \frac{\partial}{\partial y}, \quad \partial_{\perp} \equiv-\sin \Theta \frac{\partial}{\partial x}+\cos \Theta \frac{\partial}{\partial y} .
\end{aligned}
$$

Here we have defined $\tilde{\rho} \equiv \sqrt{b}(\rho-\bar{\rho})$ and $\tilde{c} \equiv c / \sqrt{b}$. In the case $c=0$, these equations are invariant under the simultaneous transformations $\Theta \rightarrow \Theta+\pi$ and $\tilde{\rho} \rightarrow-\tilde{\rho}$. The density $\rho$ is affected by $\Theta$ through the divergence term, $\partial_{\perp} \Theta=\nabla \cdot(\cos \Theta, \sin \Theta)$. The effect of this term differs for spirals with different rotation directions. The result of the numerical simulations shown in Fig. 10 elucidates the difference between $\rho$ for the two types of spirals. We infer that as $c$ increases, oscillations corresponding to one type of spiral are not allowed to exist due to the effect of the $\partial_{\perp} \Theta$ term in Eq. (13b).

\section{Conclusions}

We have proposed a mathematical model describing the evolution of spatiotemporal patterns appearing in bacteria cultures that result from the collective motion of the individual bacteria. We find that this model describes behavior in which as the bacteria population increases, the trivial uniform stationary state bifurcates sub-critically into nonuniform states exhibiting localized collective motion. Discontinuous transition representing the spontaneous formation of swarming clusters has been found in models consisting of discrete systems of self-propelled particles [25]. The validity of the continuous description adopted in our phenomenological model should be verified by studying such discrete systems. We found that as the bacteria density increases, collective motion appears throughout the entire system. In particular, assuming that the direction of the collective motion rotates gradually, two types of spirals, characterized by opposite directions of rotation, are formed. One type of 
spiral can not grow in the case that the equilibrium distribution is sufficiently asymmetric with respect to the direction of collective motions. These spatiotemporal patterns are formed through interactions between the local bacteria density and the order parameter representing the collective motion. It is an interesting problem to investigate the mathematical structures of the two types of spirals. Our study was motivated by the discovery of spatio-temporal patterns in experiments employing Proteus [12-14]. In order to construct a model that can be used to describe the morphologies observed in a wider range of systems of this type, it is necessary to add the effects of changes in bacterial population and nutrient concentration and the life cycle of cells, which were ignored here [26-28].

\section{Acknowledgements}

The author acknowledges M. Matsushita, A. Nakahara and C. Urabe for fruitful discussions, and G. C. Paquette for reading the manuscript conscientiously.

\section{A Reduction of the 2-d model}

The reductive perturbation of the 2 -d model is described below [15]. Here we assume that $\tau \ll 1$ and $\phi \sim O(\tau)$. The uniform solution, $n=n_{0}$, given in (10) and (12), satisfies the equations

$$
\begin{array}{r}
F\left\{n_{0}\right\} \equiv \rho g\left(z_{0}\right)-n_{0}+\tau \omega \frac{\partial n_{0}}{\partial \theta}=0, \\
z_{0} \equiv W_{0} e^{i(\theta-\phi)}, \quad \rho \equiv\left\langle n_{0}\right\rangle, \quad W_{0} \equiv\left\langle e^{i \theta} n_{0}\right\rangle \equiv\left|W_{0}\right| e^{i \Theta} .
\end{array}
$$

We express Eq. (8a) in terms of the above $F\{n\}$ as

$$
\tau\left(\frac{\partial n}{\partial t}+\omega \frac{\partial n}{\partial \theta}\right)=F\{n\}+\tau \hat{M} n
$$

where the operator $\hat{M}$ is defined as $\hat{M} n \equiv\left\{-\boldsymbol{v} \cdot \nabla+(\boldsymbol{v} \cdot \nabla)^{2}\right\} n$. We regard the spatial interactions as a perturbation. We expand a solution as $n=n_{0}(\rho, \Theta)+$ $\delta n$, with small $\delta n$, assuming that $\rho$ and $\Theta$ change slowly as functions of $t$ and $\boldsymbol{x}$. Equation (A.3) is approximated to lowest order in $\delta n$ and $\tau$ as

$$
\tau\left(\frac{\partial}{\partial t}+\omega \frac{\partial}{\partial \theta}\right) n_{0} \simeq \hat{L} \delta n+\tau \hat{M} n_{0}
$$

Here, $\hat{L} \delta n$ represents the part of $F\{n\}$ linear in $\delta n$. The linear operator $\hat{L}$ has the two left-eigenfunctions 1 and $\operatorname{Im} z_{0}$, because $\rho$ and $\Theta$ are arbitrary in Eq. 
(A.1). From the solvability conditions, $\langle\hat{L} \delta n\rangle=0$ and $\left\langle\operatorname{Im} z_{0} \hat{L} \delta n\right\rangle=0$, we obtain the reduced equations,

$$
\frac{\partial \rho}{\partial t}=\left\langle\hat{M} n_{0}\right\rangle \quad \text { and } \quad \frac{\partial \Theta}{\partial t}-\omega=-\frac{1}{\left|W_{0}\right|^{2}} \operatorname{Im}\left\{\left\langle z_{0} \hat{M} n_{0}\right\rangle\right\}+O(\phi) .
$$

Using Eqs. (11) and (12) for $g(z)$ and $n_{0}$, these equations become

$$
\begin{aligned}
\frac{\partial \rho}{\partial t} & =\frac{1}{2} \partial \partial^{*} \rho-\operatorname{Re}\left(\partial W_{0}\right)+\frac{1}{2} c \operatorname{Im}\left(\partial^{2} W_{0}^{2}\right)+O\left(\phi^{2}\right), \\
\frac{\partial \Theta}{\partial t} & =\omega-\frac{1}{2\left|W_{0}\right|^{2}} \operatorname{Im}\left\{W_{0}\left(\partial \partial^{*} W_{0}^{*}-\partial \rho+\frac{1}{2} \partial^{2} W_{0}\right)\right\}+c \operatorname{Re}\left(\partial W_{0}\right)+O(\phi),
\end{aligned}
$$

where we have defined $\partial \equiv \partial / \partial x-i \partial / \partial y$. The superscript ${ }^{*}$ indicates complex conjugation. As $\rho / \rho_{c}$ increases, the above equations can be approximated by Eq. (13), because $W_{0}$ approaches $e^{i \Theta} / \sqrt{b}$.

\section{References}

[1] T. Vicsek, A. Czirók, E. Ben-Jacob, I. Cohen and O. Shochet, Phys. Rev. Lett. 75 (1995) 1226.

[2] N. Shimoyama, K. Sugawara, T. Mizuguchi, Y. Hayakawa and M. Sano, Phys. Rev. Lett. 76 (1996) 3870.

[3] G. Grégoire, H. Chaté and Y. Tu, Physica D 181 (2003) 157.

[4] F. J. Nédélec, T. Surrey, A. C. Maggs and S. Leibler, Nature 389 (1997) 305.

[5] X-L. Wu and A. Libchaber, Phys. Rev. Lett. 84 (2000) 3017.

[6] U. Börner, A. Deutsch, H. Reichenbach and M. Bär, Phys. Rev. Lett. 89 (2002) 078101.

[7] E. O. Budrene and H. C. Berg, Nature 349 (1991) 630.

[8] T. Matsuyama and M. Matsushita, Critical Reviews in Microbiology 19 (1993) 117.

[9] E. Ben-Jacob, I. Cohen, A. Czirók, T. Vicsek and D. L. Gutnick, Physica A 238 (1997) 181.

[10] A. M. Lacasta, I. R. Cantalapiedra, C. E. Auguet, A. Peñaranda and L. Ramírez-Piscina, Phys. Rev. E 59 (1999) 7036.

[11] H. Shimada, T. Ikeda, J. Wakita, H. Itoh, S. Kurosu, F. Hiramatsu, M. Nakatsuchi, Y. Yamazaki, T. Matsuyama and M. Matsushita, J. Phys. Soc. Jpn. 73 (2004) 1082. 
[12] Y. Shimada, A. Nakahara, M. Matsushita and T. Matsuyama, J. Phys. Soc. Jpn. 64 (1995) 1896.

[13] A. Nakahara, Y. Shimada, J. Wakita, M. Matsushita and T. Matsuyama, J. Phys. Soc. Jpn. 65 (1996) 2700.

[14] K. Watanabe, J. Wakita, H. Itoh, H. Shimada, S. Kurosu, T. Ikeda, Y. Yamazaki, T. Matsuyama and M. Matsushita, J. Phys. Soc. Jpn. 71 (2002) 650 .

[15] Y. Kuramoto, Chemical Oscillations, Waves, and Turbulence (Dover, New York, 2003).

[16] C. Allison, H-C Lai, D. Gygi and C. Hughes, Mol. Microbiol. 8 (1993) 53.

[17] O. Rauprich, M. Matsushita, C. J. Weijer, F. Siegert, S. E. Esipov and J. A. Shapiro, J. Bacteriol. 178 (1996) 6525.

[18] T. Matsuyama, Y. Takagi, Y. Nakagawa, H. Itoh, J. Wakita and M. Matsushita, J. Bacteriol. 182 (2000) 385.

[19] H. C. Berg and D. A. Brown, Nature 239 (1972) 500.

[20] H. Y. Lee and M. Kardar, Phys. Rev. E 64 (2001) 056113.

[21] R. A. Simha and S. Ramaswamy, Phys. Rev. Lett. 89 (2002) 058101.

[22] I. S. Aranson and L. S. Tsimring, Phys. Rev. E 71 (2005) 050901.

[23] E. Ben-Jacob, I. Cohen, O. Shochet, A. Tenenbaum, A. Czirók and T. Vicsek, Phys. Rev. Lett. 75 (1995) 2899.

[24] Competition between spirals with different rotation directions is also known for liquid crystal. T. Frisch, S. Rica, P. Coullet and J. M. Gilli, Phys. Rev. Lett. 72 (1994) 1471.

[25] G. Grégoire and H. Chaté, Phys. Rev. Lett. 92 (2004) 025702.

[26] A. Czirók, M. Matsushita and T. Vicsek, Phys. Rev. E 63 (2001) 031915.

[27] B. P. Ayati, J. Math. Biol. 52 (2006) 93.

[28] B. P. Ayati, preprint, arXiv:q-bio.CB/0507027. 